\title{
Einleitung: Das Softwareprojekt bei der Muster GmbH
}

Die Muster $\mathrm{GmbH}^{1}$ ist ein mittelständisches deutsches Unternehmen mit zwei zusätzlichen internationalen Niederlassungen. Das Unternehmen besitzt zum einen eine Produktionsfirma in der Tschechischen Republik und zum anderen eine Vertriebsfirma in den USA.

Die Muster GmbH beabsichtigt, ab dem nächsten Jahreswechsel ein neues Finanzbuchführungssystem inkl. Kostenrechnung produktiv einzusetzen.

Die Steuerdeklaration soll nicht in das eigene Haus verlagert werden. Hier soll es bei der bisherigen Arbeitsteilung mit dem Steuerberater bleiben.

Die Software wird nicht von den internationalen Niederlassungen eingesetzt werden. Es ist jedoch zwingend notwendig, dass das Controlling der drei Standorte zusammenfließt.

Zur Einführung der neuen Software soll ein Projekt aufgesetzt werden. Für die Implementierung werden vier Monate veranschlagt.

In einem Vorprojekt waren bereits das Lastenheft erstellt und die Software-Auswahl getroffen worden. Anhand eines strukturierten Verfahrens war die Wahl auf das Finanzbuchführungssystem des Anbieters Laedi Software gefallen.

Dem Projekt werden drei Mitarbeiter mit jeweils $40 \%$ ihrer täglichen Arbeitszeit zugeteilt. Herr Itzel stammt aus dem Bereich IT, Frau Kaufmann aus dem Bereich kaufmännische Verwaltung und Herr Leiter aus der Geschäftsleitung. Herr Leiter soll die interne Projektleitung übernehmen.

Für externe Projektunterstützung (z. B. für Schulung und Training) steht ein Budget von $70 \mathrm{~T} €$ zur Verfügung.

\footnotetext{
${ }^{1}$ Die Muster GmbH ist ein fiktives Unternehmen. Alle in diesem Buch genannten Namen von Unternehmen, Mitarbeitern und Software-Lösungen sind zufällig gewählt. Etwaige Übereinstimmungen mit real existierenden Unternehmen, Personen und Produktnamen sind nicht beabsichtigt.
} 